\title{
An analysis of balance velocities over the Greenland ice sheet and comparison with synthetic aperture radar interferometry
}

\author{
J. L. Bamber, ${ }^{1}$ R. J. Hardy, ${ }^{1}$ I. Joughin ${ }^{2}$ \\ ${ }^{1}$ Bristol Glaciology Centre, School of Geographical Sciences, University of Bristol, Bristol, BSo 1SS, England \\ ${ }^{2}$ Fet Propulsion Laboratory, California Institute of Technology, Pasadena, California 91109, U.S.A.
}

\begin{abstract}
Balance velocities for the Greenland ice sheet have been calculated from a new digital elevation model (DEM), accumulation rates and an existing ice-thickness grid, using a fully two-dimensional finite-difference scheme. The pattern of velocities is compared with velocities derived from synthetic-aperture radar (SAR) interferometry for three different regions of the ice sheet. Differences between the two estimates of velocity highlight the respective strengths and weaknesses of the datasets and techniques used. A comparison with ten global positioning system-derived velocities indicates that the balance-velocity scheme and input datasets used here provide a remarkably good representation of the velocity distribution inland from the margins. These balance-velocity data, therefore, could help constrain numerical ice-sheet models. The balance velocities were found to be unreliable close to the ice-sheet margins due to larger errors in ice thickness, surface slope and ablation rate in this region. Comparison of the balance velocities with SAR interferometry in the region of the "Northeast Greenland Ice Stream" indicates the importance of the smoothing distance that must be applied to the DEM before calculating balance velocities. A smoothing distance of 20 times the ice thickness gave good agreement between the two measures of velocity.
\end{abstract}

\section{NOTATION}

$B \quad$ Baseline separation

$U_{\mathrm{b}} \quad$ Balance velocity

$U_{\mathrm{bf}}$ Balance velocity calculated using flowline methodology

$U_{\text {b10 }}$ Balance velocity calculated with 10 ice-thickness smoothing

$U_{\mathrm{b} 20}$ Balance velocity calculated with 20 ice-thickness smoothing

$U_{\mathrm{ds}}$ Downslope velocity from InSAR data

$U_{\text {los }}$ Horizontal line-of-sight velocity from InSAR data

$Z \quad$ Ice thickness

$\phi \quad$ Angle between SAR look direction and direction of ice motion

\section{INTRODUGTION}

An understanding of the dynamic behaviour of the Antarctic and Greenland ice sheets is crucial to our ability to model their past and future evolution, especially if models are going to be used to predict changes in mass balance due to global climate change. Aspects of the dynamics are, however, not well understood and are inadequately measured due to a lack of observations and difficulties in modelling the dynamics. This is largely a result of uncertainties in several key variables and physical processes such as the rate factor in the flow law of ice and basal sliding behaviour. Nonetheless, a number of numerical models have been used to investigate the dynamics of the Greenland ice sheet
(Huybrechts and others, 1991; Letréguilly and others, 1991a; Greve, 1997). These models have been used to simulate the ice-sheet geometry, temperature and velocity fields. Such schemes, in general, operate on a relatively coarse resolution (typically $20 \mathrm{~km}$ ) and are often limited by their use of the shallow-ice approximation, which assumes that longitudinal stresses are averaged out over distances of 10-20 times the ice thickness, $Z$ (Paterson, 1994). This means that they do not accurately capture the fine-scale detail (i.e. $<10-20 Z$ ) where longitudinal stresses are important.

These large-scale models have been used either to simulate past climatic reconstructions (Letréguilly and others, 1991b) or to predict the effect of global warming on the ice sheet (Huybrechts and others, 1991). Perhaps of greatest concern for this latter application is the need to employ a variable enhancement factor (typically about $0.1-10$ ) in the flow law of ice due to uncertainty in the ice rheology. Furthermore, uncertainty in estimating the basal friction means that an additional adjustable parameter is used in the sliding law. Measurements of ice motion can potentially provide a valuable constraint for numerical models (Bamber and Huybrechts, 1996). In situ measurements are sparse, however, and remote-sensing methods have begun to provide much-needed data. In particular, direct measurement of the short-term surface velocity can be made either from displacement of features observed in pairs of visible images (feature tracking) (Scambos and others, 1992) or by interferometric synthetic-aperture radar (InSAR) techniques (e.g. Joughin and others, 1995, 1996a). In North Greenland, for example, data from crossing ascending and descending 
satellite overpasses have recently been used to measure the three-dimensional velocity field for Ryder Gletscher (Joughin and others, 1998) and Storstrømmen (Mohr and others, 1998). Feature-tracking methods using visible imagery are limited to fast-moving regions where there are sufficient features to track, and InSAR methods require some form of ground control, albeit at a limited number of locations. Other problems with the use of InSAR will be discussed later.

Another estimate of the velocity field, called balance velocities, can be obtained by integrating the ice flux from the ice divide to the margins (e.g. Budd and Carter, 1971; Paterson, 1994; Budd and Warner, 1996). For this calculation, surface-topography, ice-thickness and accumulation data are required. With the aid of these data it is possible to calculate balance velocities over the whole Greenland ice sheet. Balance velocities are, however, a derived quantity, and various assumptions are made in their derivation. In this paper we examine the relative strengths and weaknesses of InSAR and balance velocities as tools for investigating the velocity field of ice sheets.

\section{VELOGITY DATASETS}

\section{Balance velocities}

The balance velocity $\left(U_{\mathrm{b}}\right)$ is a depth-averaged value necessary to maintain the steady state of an ice sheet assuming that it is in balance. This implies that the outward flux exactly matches the net accumulation (minus the ablation), so that there is no change in ice thickness, $Z$, with time. A continuity equation is then derived, and by integrating the net accumulation from the summit to the ice edge, the mass flux at any point can be estimated. The balance velocity can then be calculated if the ice thickness is known.

One of the advantages of balance velocities is that they provide a synoptic-scale estimate of velocity, which may be useful in constraining numerical models. They have also been used in a number of previous studies for a variety of applications (cf. Budd and Carter, 1971; Radok and others, 1982; Budd and Warner, 1996; Joughin and others, 1997). They represent, however, a depth- and time-averaged quantity. The time constant for the dynamic response of a drainage basin of the Greenland ice sheet is typically on the order of 1000 years (Paterson, 1994), whereas the accumulation and ablation data have been obtained over a much shorter time-scale of 20-50 years. The implicit assumption made here is that accumulation and ablation rates have remained relatively constant during the dynamic response time of the basin. Based on the results from the two Greenland summit cores, GRIP and GISP2, this seems a reasonable assumption (Grootes and others, 1993). Balance velocities are thus representative of the mean depth-averaged flow over a period on the order of 1000 years. Measured velocities, however, are usually short-term surface values subject to possible variability due, for example, to changes in basal lubrication (Joughin and others, 1996b).

It is also necessary to consider the method used for calculating $U_{\mathrm{b}}$. Typically, a flux-gate approach has been used. Here we have adopted a two-dimensional finite-difference scheme that does not require an arbitrary gate width to be specified (Budd and Warner, 1996). Perhaps more important, however, is the smoothing applied to the elevation data. This is necessary to ensure that the ice flows downhill and that the effects of longitudinal stresses have been averaged out. Removing the effects of longitudinal stresses in this way is known as the shallow-ice approximation. It is believed that a smoothing distance of $10-20 Z$ is required (Paterson, 1994). The true scale over which longitudinal stresses can be ignored, however, is a function of the ice rheology and geometry, which are spatially variable quantities. This issue is explored further when comparing balance velocities with InSAR-derived velocities.

One of the strengths of balance-velocity estimates is that the state of imbalance is, in general, much less than the uncertainty in assessing the deformation and sliding properties of ice (Van der Veen and Whillans, 1990). Consequently, balance velocities and fluxes could provide a useful guide for dynamics where direct observations are sparse (Bamber and Huybrechts, 1996; Budd and Warner, 1996). However, the accuracy of the calculated balance velocity depends upon the quality of the input data and the method of spatial discretization. Balance velocities represent the true depthaveraged velocity if, and only if, (i) there are no errors in the input datasets, (ii) the drainage basin is in balance, (iii) there is steady-state, time-invariant flow and (iv) the ice flow obeys the dynamical assumptions used in the calculation of $U_{\mathrm{b}}$. The relative importance of these factors will be illustrated by a comparison with (nearly) instantaneous InSAR-derived surface velocities and global positioning system (GPS) data.

Previous methodologies to calculate $U_{\mathrm{b}}$ have generally applied a flowline or flux-gate approach for spatial discretization. In this study, a two-dimensional finite-difference scheme (Budd and Warner, 1996) has been used. The differences between balance velocities calculated here and a recently published flowline approach (Joughin and others, 1997) are discussed when comparing the balance velocities with InSAR measurements for the "Northeast Greenland Ice Stream". As mentioned earlier, however, the smoothing applied to the digital elevation model (DEM) is probably more important than the methodology adopted for calculating $U_{\mathrm{b}}$. This is also discussed later.

\section{Input data for calculating $U_{\mathrm{b}}$}

Three datasets are required: a DEM to determine the direction of flow; ice thickness; and net ice input (accumulation minus ablation). The DEM has been derived primarily from analysis and interpretation of satellite radar-altimeter data from the geodetic phase of the European remote-sensing satellite ERS-1 and is described elsewhere (Bamber and others, 1997). A $2.5 \mathrm{~km}$ resolution DEM of the Greenland ice sheet was produced for this application (Fig. 1). The height measurements in the DEM were validated using a set of airborne laser-altimeter flight-lines over southern Greenland and indicate that the radar-altimeter data have an accuracy of approximately 1-10 $\mathrm{m}$ depending on the regional slope (Bamber and others, 1998). Where no valid radar-altimeter data were available, the DEM was supplemented with data from a previously published DEM of Greenland, which incorporated stereo photogrammetric and cartographic datasets around the ice-sheet margins and for land areas (Ekholm, 1996).

The shallow-ice approximation is based on the assumption that on a scale of $10-20 Z$, flow is in the direction of the surface slope (Paterson, 1994). As a result, the DEM was smoothed on a spatially dependent scale of $10 Z$ and $20 Z$, producing two balance-velocity grids denoted $U_{\mathrm{b} 10}$ and $U_{\mathrm{b} 20}$, respectively. The smoothing was applied to ice thick- 
nesses of $>500 \mathrm{~m}$. Below this value, a fixed smoothing over two gridcells (i.e. $5 \mathrm{~km}$ ) was used. Two smoothing distances were chosen to investigate how this affected the results.

The ice-thickness data were obtained from airborne radio-echo sounding measurements with a variable track spacing of about 50-100 km (Letréguilly and others, 1991b). The error in an individual measurement is on the order of $10 \%$. Errors will be greater than this for gridpoints lying between flight tracks or beyond the limit of coverage near the margins, where there was sparse coverage.

The net ice input is the accumulation minus the ablation. The accumulation grid was derived from a recent, unpublished map of accumulation over the ice sheet by $\mathrm{F}$. Jung-Rothenhäusler and others (in press). For brevity, this will be referred to as the J-R dataset. The uncertainty in the accumulation dataset is quoted as $10 \%$. Ablation has been calculated using a positive degree-day approach using a single degree-day factor of $8 \mathrm{~mm}^{\circ} \mathrm{C}^{-1} \mathrm{~d}^{-1}$ (Huybrechts and others, 1991). The uncertainty in ablation, using this approach and a single degree-day factor, is estimated to be $20 \%$ (personal communication from R.J. Braithwaite, 1999).

\section{InSAR measurements of velocity}

The use of InSAR to derive surface velocities over ice is now well established (Joughin and others, 1995; Rignot and others, 1995), and only the most relevant points are summarized here. The repeat-pass method requires two images, collected at almost identical orbital locations (within several hundred metres) but in different epochs. A complex interferogram is formed as the product of one image with the complex conjugate of a second. The interference pattern depends primarily on the topography and any displacement between the two epochs. The separation of the satellite for the two measurements (the baseline separation, $B$ ) determines the sensitivity of the topographic term. Another factor, however, that influences the interferometric phase pattern is variation in electromagnetic path length introduced by the atmosphere (in particular, the presence of fronts associated with water-vapour variations) (Hanssen and others, 1998). There are various ways to separate the motion and topography, using either an independent DEM or multiple interferograms (Joughin and others, 1995). Errors in the baseline derived from orbital-state vectors result in a nearly linear phase ramp in the interferogram. Baseline errors are reduced by using ground-control points (e.g. in situ GPS measurements and/or a DEM) to solve for the baseline.

An interferogram by itself provides only a measure of relative motion. Tie points of known velocity and elevation are needed to calibrate the data in order to remove baseline errors and provide absolute reference. Here, two different approaches have been taken depending on the availability of tie points. Where exposed bedrock is present, this has been used to provide zero-motion tie points. Elsewhere, balance velocities (calculated from a flowline procedure (Joughin and others, 1997)) were used as velocity tie points in areas of slow flow. A maximum velocity threshold of $12 \mathrm{~m} \mathrm{a}^{-1}$ was used.

In this study, three InSAR datasets were used. Their locations are shown by the polygons in Figure 1 . The first two datasets cover Humboldt Gletscher and Petermann Gletscher, in northwestern Greenland. For the northern sec-

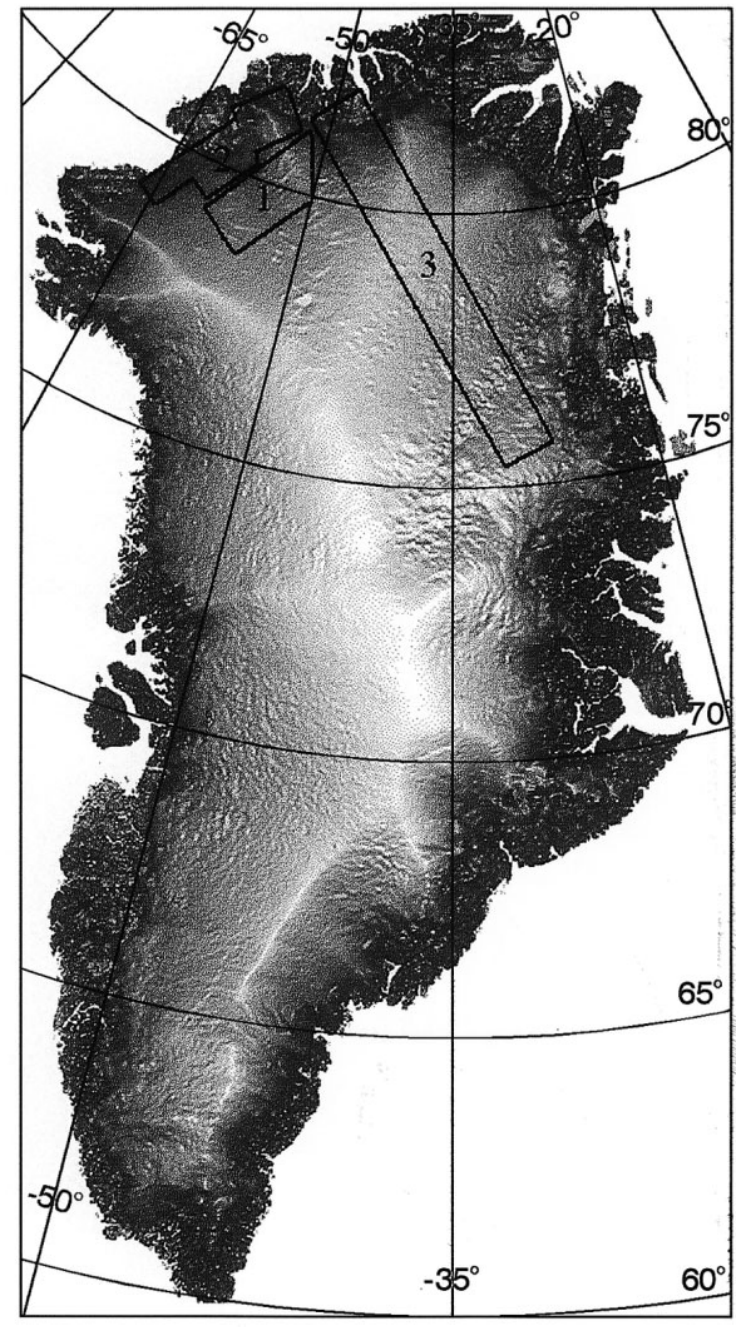

Fig 1. A $2.5 \mathrm{~km}$ resolution DEM of the Greenland ice sheet produced from the 336 days of the geodetic phase of ERS-1 (Bamber and others, 1997). The numbered polygons show the locations of the InSAR data used in this study.

tor (2), the velocity was estimated to be accurate to $2.3 \mathrm{~m} \mathrm{a}^{-1}$ (Joughin and others, 1996a). In this case, it was possible to use bedrock near the coast as zero-motion tie points. For sector 1, balance velocities were used to give an absolute velocity estimate, as no bedrock was within the scenes. The third dataset was a $785 \mathrm{~km}$ long strip stretching from the central part of the North Greenland coastline towards the east coast. It covered the inland part of the Northeast Greenland Ice Stream, recently identified from SAR imagery (Fahnestock and others, 1993). The strip was tandem phase data (where the two satellites, ERS-1 and ERS-2, were in orbits separated by 1 day) acquired during March 1996. It extended as far as the east coast, but, due to a phase "spike", an unexplained phase gradient, phase continuity was not maintained beyond the southern limit indicated by sector 3. Due to the absence of bedrock inland, absolute velocities were obtained using balance velocities (with values of $<12 \mathrm{~m} \mathrm{a}^{-1}$ ) as tie points. In all cases, the horizontal line-of-sight velocity estimate $\left(U_{\mathrm{los}}\right)$ was converted to a downslope value $\left(U_{\mathrm{ds}}\right)$ using slope information from the DEM. A comparison of a similar InSAR dataset with GPS measurements gave rms error estimates of $<4 \mathrm{ma}^{-1}$ (Joughin and others, 1998) for $U_{\mathrm{ds}}$.

The three InSAR datasets provide coverage of several different flow regimes over the ice sheet: fast flow near the margins where the ice is flowing into outlet glaciers, part of 
the Northeast Greenland Ice Stream, as well as a large area of relatively slow flow $\left(0-50 \mathrm{~m} \mathrm{a}^{-1}\right)$ in the interior. In the interior, however, it is important to note that the InSAR-derived velocities were tied to balance velocities in slow-moving areas.

$U_{\mathrm{ds}}$ is a measure of the surface velocity. $U_{\mathrm{b}}$ is an estimate of the depth-averaged velocity. To compare the two it is necessary to convert $U_{\mathrm{b}}$ to a surface velocity, or $U_{\mathrm{ds}}$ to a depthaveraged value. The ratio of depth-averaged to surface velocity depends primarily on the temperature profile in the ice. It typically varies between 0.8 and 1.0 (Paterson, 1994). Here the ratio was obtained from the output of a three-dimensional thermomechanical model (Huybrechts and others, 1991). The model was run in a steady-state mode, where the ice thickness is kept fixed and the model physics is in equilibrium with the ice-sheet geometry and massbalance data (personal communication from P. Huybrechts, 1998). The model has 14 layers. The depth-averaged velocity is the mean over these 14 layers, and the ratio was obtained by dividing this mean value by the surface velocity (layer 1). The mean ratio for the whole ice sheet was 0.925 . The spatial pattern of the ratio is plotted in Figure 2.

\section{GPS velocity data}

Ten GPS velocity measurements, collected as part of a mass-

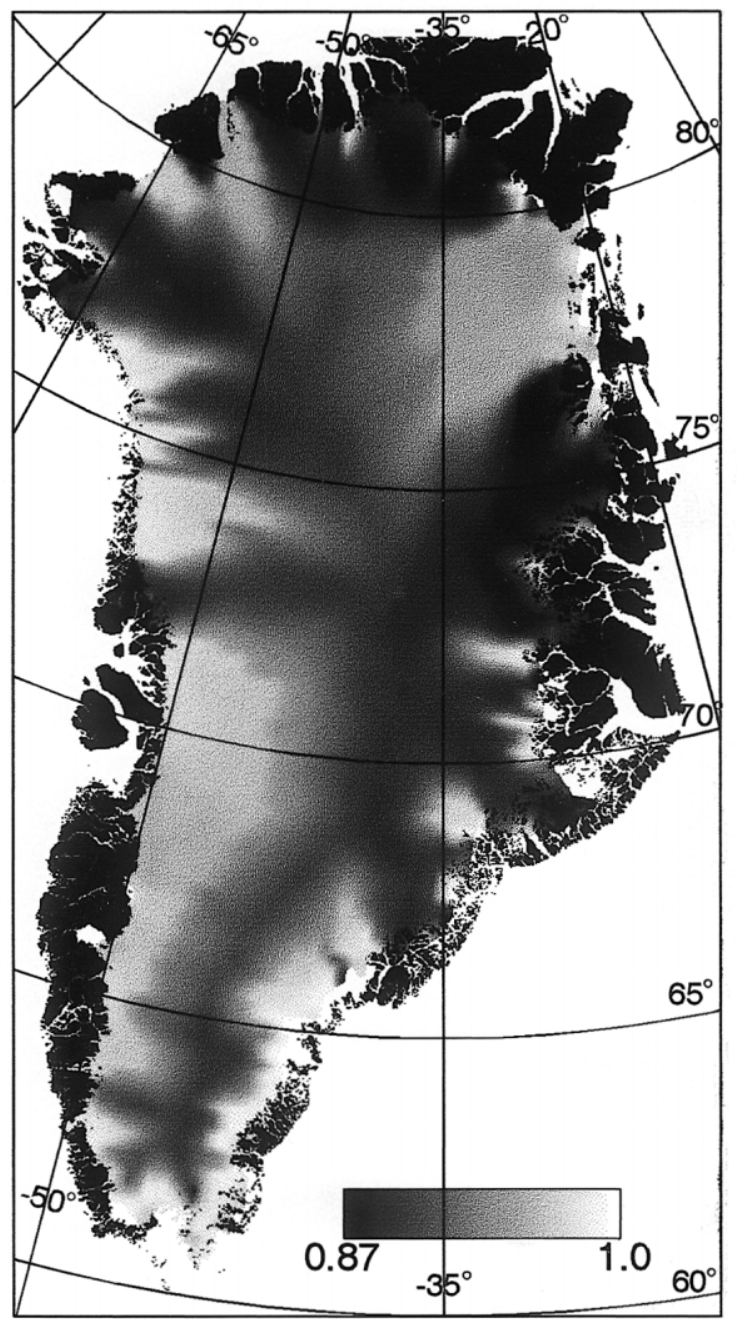

Fig 2. The ratio of depth-averaged velocity to surface velocity, obtained from simulations of a three-dimensional thermomechanical ice-sheet model (personal communication from $P$. Huybrechts, 1997). balance experiment by the Byrd Polar Research Center, Ohio State University, were available for comparison with the balance velocities. Horizontal velocities at the sites ranged from 2 to $178 \mathrm{~m} \mathrm{a}^{-1}$, and the errors in these estimates are negligible (about $1-2 \mathrm{~cm} \mathrm{a}^{-1}$ ). The data were collected during three field seasons between the summers of 1995 and 1997 (personal communication from G. Hamilton, 1998). The velocities were calculated from measurements of position taken approximately 1 year apart. The locations of the GPS points are indicated in Figure 3 by white crosses.

\section{RESULTS AND DISGUSSION}

The balance-velocity pattern over the ice sheet is shown in Figure 3. A comprehensive, detailed view of the velocity field is displayed, based on the assumption of steady-state, balanced conditions.

The balance velocities clearly delineate the pattern of flow in each drainage basin. The major ice divides are visible as areas of very low horizontal flow. All the main outlet glaciers and their catchment areas can be seen. Particularly striking is the large ice stream in the northeast, previously identified in SAR imagery (Fahnestock and others, 1993). A

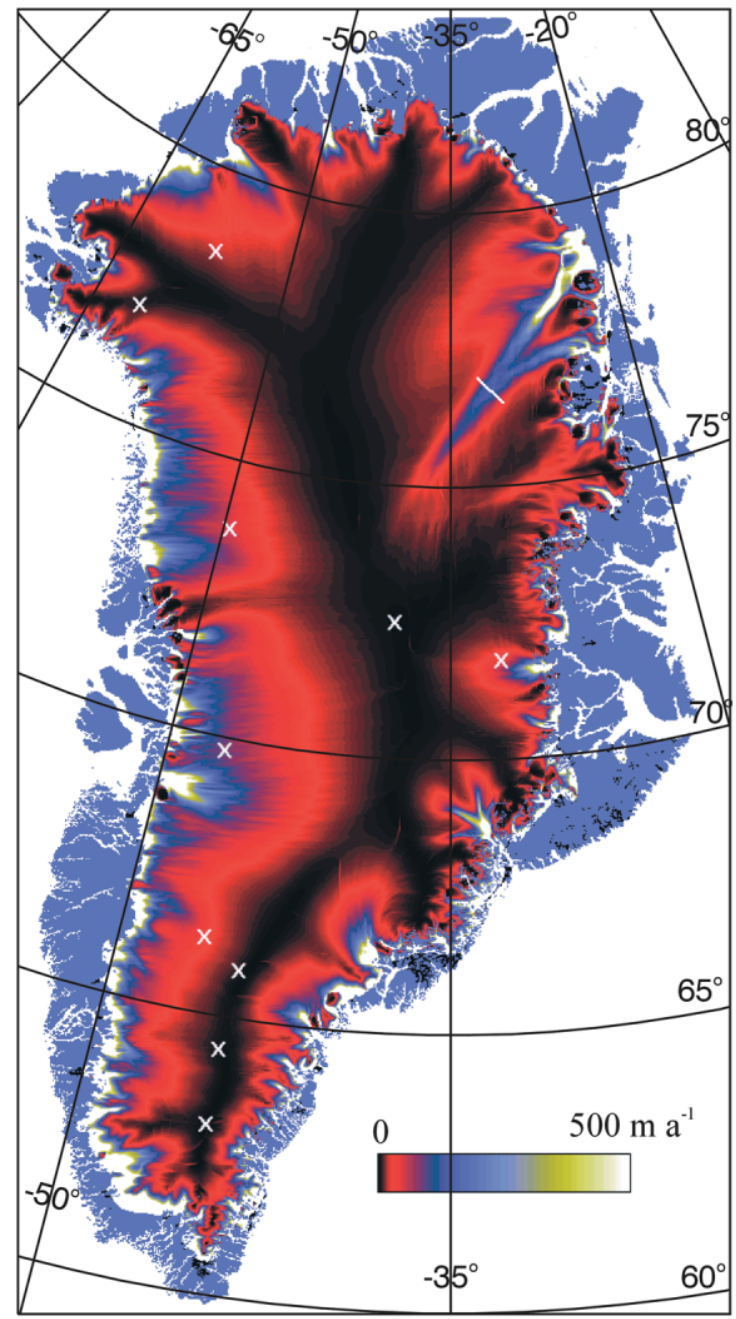

Fig 3. Calculated balance velocities over the Greenland ice sheet obtained using the two-dimensional computational scheme of Budd and Warner (1996). The locations of the GPS data used in this study are shown by crosses. The line running perpendicular to the Northeast Greenland Ice Stream indicates the location of several velocity profiles presented in Figure 7. 
more detailed investigation of the balance velocities in this region is given in the next section.

There are some other obvious features relating to the general pattern of flow. On the west coast the flow appears to be largely unconstrained by bedrock relief. This has resulted in generally less well-defined outlet glaciers along most of the northwest coastline. By contrast, much of the rest of the ice sheet is dominated by a series of flow units with well-defined outlet glaciers and catchment areas which must reflect a fundamental difference between the continental margins on the west coast and those elsewhere. It should be noted that velocities close to the coast are less reliable, due to larger errors in the ice-thickness, DEM and ablation estimates in this region (discussed later). It is still possible, however, to place confidence in the pattern of drainage displayed and the relative contribution of different areas to the total flux at the margin.

\section{Comparison with the GPS data}

Table 1 provides a comparison between $U_{\mathrm{b}}$ (corrected from a depth-averaged to a surface value) and GPS-derived velocities. Included here are values of balance velocity calculated using the finite-difference scheme described here and a flowline method $\left(U_{\mathrm{bf}}\right)$ (Joughin and others, 1997). Bilinear interpolation was used to calculate the balance velocity at the same location as the GPS measurements. With only ten GPS points it is difficult to draw statistically meaningful conclusions. The agreement between $U_{\mathrm{b} 20}$ and the GPS data, however, is surprisingly good. The mean difference is $4.4 \mathrm{~m} \mathrm{a}^{-1}$ with a standard deviation of $9.4 \mathrm{~m} \mathrm{a}^{-1}$. Removing the Jakobshavn catchment point brings these values down to $1.8 \pm 4.7 \mathrm{~m} \mathrm{a}^{-1}$ (or $6.5 \pm 16.8 \%$ ). This provides support for the methodology adopted here.

Table 1. Comparison between GPS and balance-velocity estimates

\begin{tabular}{lccrrr}
\hline Site & Latitude & Longitude & $\begin{array}{c}\text { GPS } \\
\text { velocity }\end{array}$ & $U_{\mathrm{b} 20}$ & $U_{\mathrm{bf}}$ \\
& ${ }^{\circ} \mathrm{N}$ & ${ }^{\circ} \mathrm{W}$ & $\mathrm{m} \mathrm{a}^{-1}$ & $\mathrm{~m} \mathrm{a}^{-1}$ & $\mathrm{~m} \mathrm{a}^{-1}$ \\
& & & & & \\
\hline South Dome & 63.150 & -44.823 & 3.2 & 0.2 & 0.7 \\
Saddle & 65.999 & -44.499 & 5.3 & 5.9 & 5.7 \\
Dye-2 & 66.498 & -46.273 & 27.4 & 35.7 & 32.2 \\
Jakobshavn catchment & 69.060 & -46.829 & 178.0 & 206.0 & 293.1 \\
Crawford Point & 69.881 & -46.979 & 86.3 & 93.4 & 67.4 \\
Summit/GISP2 & 72.575 & -38.456 & 1.9 & 1.4 & 1.7 \\
Daugaard-Jensen Gletscher & 71.887 & -32.057 & 58.0 & 52.1 & 60.1 \\
NASA/U & 73.842 & -49.496 & 45.7 & 50.6 & 40.8 \\
Camp Century & 77.139 & -61.039 & 4.2 & 5.2 & 6.0 \\
Humboldt Gletscher & 78.526 & -56.832 & 17.4 & 21.2 & 21.9
\end{tabular}

* Greenland Ice Sheet Project II.

\section{Comparison between balance velocities and InSAR data}

The results are illustrated in Figure $4 a-d$.

\section{The $785 \mathrm{~km}$ strip}

Over much of the slower-moving central part of sector 3 the agreement between $U_{\mathrm{b} 20}$ and $U_{\mathrm{ds}}$ is within about $5 \mathrm{~m} \mathrm{a}^{-1}$. It should be noted that balance velocities were used as tie points for the InSAR data in this region. About 100-250 km from the northern end of the strip is an area with large relative and
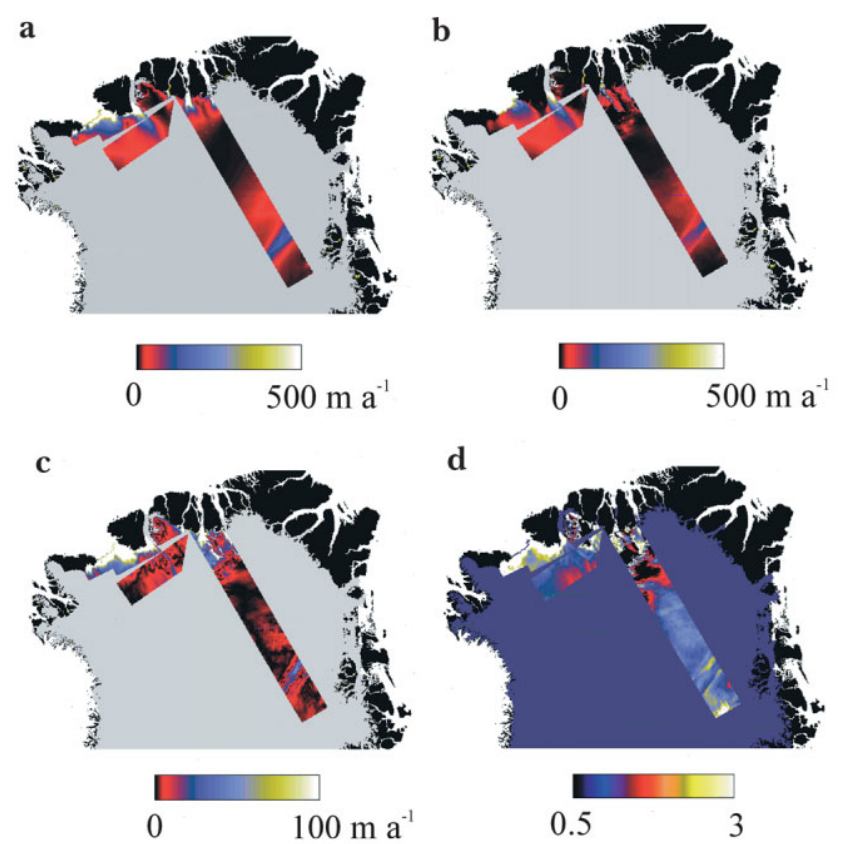

Fig 4. (a) The balance velocities $\left(U_{\mathrm{b} 20}\right)$ for the same geographical location as the InSAR coverage. (b) The InSAR velocities corrected to account for look direction and the difference between surface and depth-averaged velocities. (c) The absolute difference between the InSAR velocities and $U_{\mathrm{b} 20}$. (d) The ratio of $U_{\mathrm{b} 20}$ to the InSAR velocities. The background colour over the ice sheet represents a ratio of 1 (i.e. perfect agreement).

absolute differences, however. This is because the direction of flow is almost perpendicular to the satellite look direction in this region, resulting in a large sensitivity to errors in both $U_{\text {los }}$ and the angle between the look direction and the direction of flow $(\phi)$. Figure 5 shows the error in downslope velocity as a function of $\phi$ for a fixed phase error of $\pi \mathrm{rad}$ in $U_{\text {los. }}$ Results for the three repeat periods relevant to the ERS mission are plotted. The lines in Figure 5 were calculated based on the assumption that each fringe in an interferogram represents $7.6 \mathrm{~cm}$ horizontal displacement. The graph in Figure 5 does not take into account errors in $\phi$, which are difficult to estimate and, depending on the local flow conditions, highly variable at shorter wavelengths (see discussion below).

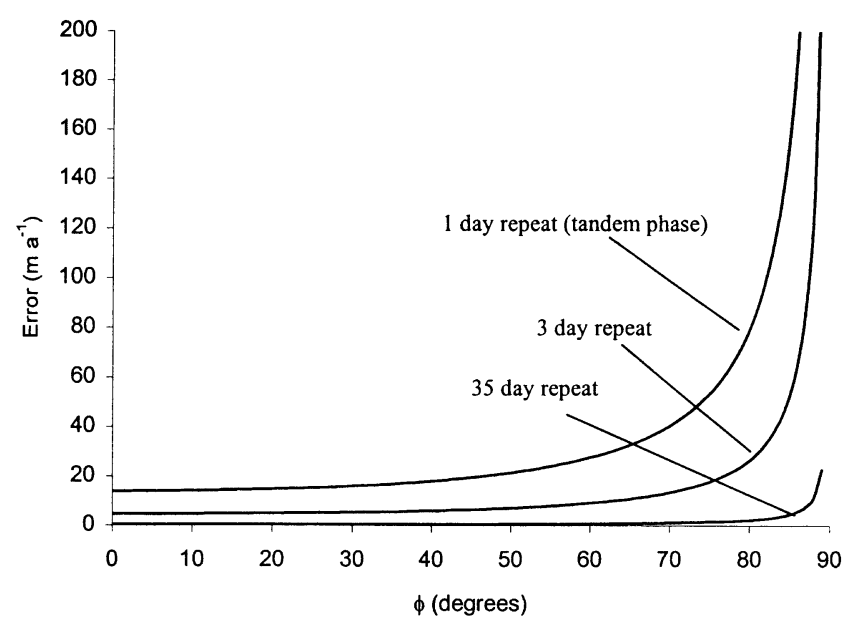

Fig. 5. Plot of the errors in the line-of-sight velocity as a function of the angle $\phi$ between the SAR look direction and the ice-flow direction. The error has been plotted for the three time intervals relevant to the ERS missions. 
The error in $U_{\mathrm{ds}}$ exceeds $20 \mathrm{ma}^{-1}$ at $47^{\circ}$ for tandem phase data and $78^{\circ}$ for 3 day repeat data. This particular error can be minimized with the use of 35 day repeat data. There are, however, other factors (such as loss of coherence between the two scenes and modulo $2 \pi$ phase ambiguities due to the displacement producing phase changes exceeding $2 \pi$ ) that limit the use of this longer repeat period in glaciological applications. A thorough treatment of the other errors associated with the InSAR measurement principle has been provided elsewhere (Joughin and others, 1996a). With adequate ground control, $U_{\text {los }}$ can be obtained with an accuracy of a few metres per year. The conversion from $U_{\text {los }}$ to $U_{\mathrm{ds}}$ introduces a further error, however, due to uncertainties in $\phi$. Furthermore, atmospheric effects can introduce phase shifts of about $180^{\circ}$ which are also difficult to account for and remove (Hanssen and others, 1998).

The other area of poor agreement, in absolute (but not relative) terms, is in the vicinity of the Northeast Greenland Ice Stream. Examination of the DEM in this region (Fig. 6) indicates that the flow is strongly influenced by longitudinal stresses, so the choice of smoothing distance required to remove the effects of longitudinal stresses is particularly important. An unsmoothed shaded isometric plot looking down the ice stream is shown in Figure 6a together with smoothed versions in Figure $6 \mathrm{~b}$ and c. Using a smoothing distance of $10 Z$, two "channels" bounding the ice stream are visible (Fig. 6b), and the finite-difference scheme places the flow partly in these channels. If a smoothing distance of $20 Z$ is used, the bifurcation of the flow does not occur and there is an almost constant ratio between $U_{\mathrm{b} 20}$ and $U_{\mathrm{ds}}$. A graph of velocity profiles across the flow is presented in Figure 7, demonstrating, quantitatively, the behaviour described above. The location of the profiles is marked by the white line in Figure 3. $U_{\mathrm{b} 20}$ and $U_{\mathrm{b} 10}$ are shown alongside the fully corrected InSAR data. In the $10 Z$ smoothing case, the ice flux is concentrated in the northern channel at the boundary of the ice stream and near the centre. Using a $20 Z$ smoothing factor, this no longer occurs and the general shape of the profile matches the interferometry well. The level of agreement can also be seen in Figure 4d. There is better agreement throughout the southern half of sector 3 but especially in the region of the ice stream, using a smoothing distance of $20 Z$.

Figure 8 shows the spatial pattern of differences between $U_{\mathrm{b} 20}$ and $U_{\mathrm{b} 10}$. Positive differences occur when $U_{\mathrm{b} 10}$ is greater than $U_{\mathrm{b} 20}$. The mass flux in both cases is the same, so areas of positive differences must be associated with nearby areas of negative differences. This is most easily seen around the Northeast Greenland Ice Stream. Here, the $U_{\mathrm{b} 10}$ velocity field generally concentrates flow at the margins of

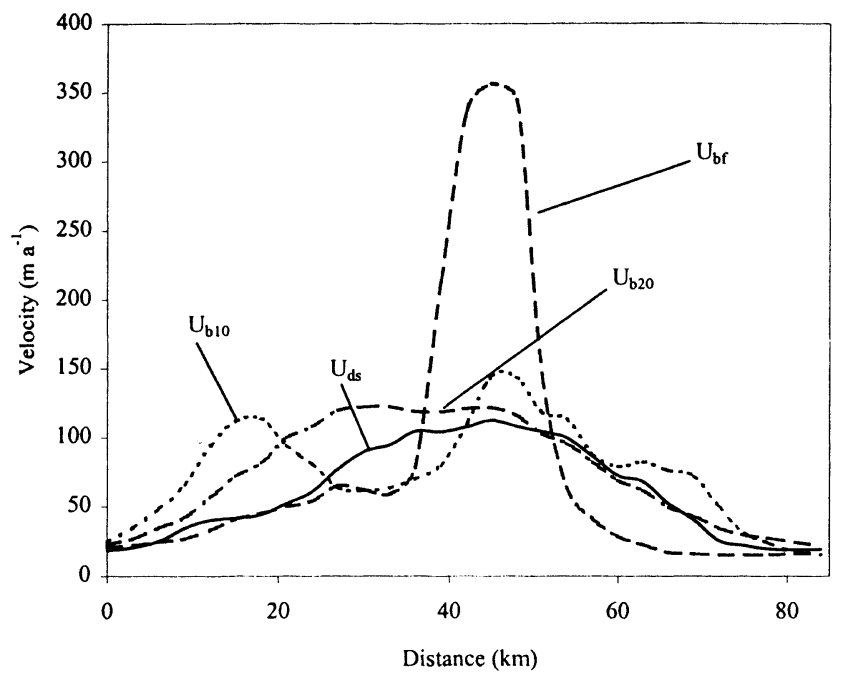

Fig. 7. Plot of velocity profiles across the Northeast Greenland Ice Stream. The location of the profile is indicated by the white line in Figure 3. Balance-velocity profiles are plotted for two different smoothing distances (10Z and 20Z) and using a flowline approach (Joughin and others, 1997). The InSARderived velocity data are also plotted for comparison.

the stream, in "channels" clearly visible in Figure 6b. There is an associated reduction in velocity estimate in the central part of the ice stream. Similar behaviour, where the flow is split into a larger number of narrower, faster-flowing "channels", occurs for several other outlet glaciers for the $U_{\mathrm{b} 10}$ velocity field.

The flowline method for deriving $U_{\mathrm{b}}$, used by Joughin and others (1997), is also shown in Figure 7. It tends to concentrate flow too closely to the centre of the ice stream. Comparison between $U_{\mathrm{b} 20}$ and $U_{\mathrm{bf}}$ suggests that this effect occurs for other outlet glaciers and fast-flow features on the ice sheet. In the flowline method a fixed smoothing distance of $20 \mathrm{~km}$ was used on the DEM. The problem was attributed to smoothing introduced by the radar altimeter (used to derive the DEM) and to the processing employed. Figure $6 \mathrm{a}$ indicates that the former effect (altimeter smoothing) is not the problem. The ice thickness in this region is about $2000 \mathrm{~m}$, indicating that the smoothing distance used to produce $U_{\mathrm{b} 10}$ and Figure $6 \mathrm{~b}$ is close to the $20 \mathrm{~km}$ fixed value used by Joughin and others (1997). $U_{\mathrm{b} 10}$ and $U_{\mathrm{bf}}$, plotted in Figure 7, show different behaviour, however. It seems likely, therefore, that the increased velocities in the centre of the feature resulted, not from the smoothing applied to the DEM, but from another aspect of the methodology.

The discrepancy between $U_{\mathrm{b} 20}$ and $U_{\mathrm{ds}}$ in the region of the Northeast Greenland Ice Stream highlights a problem
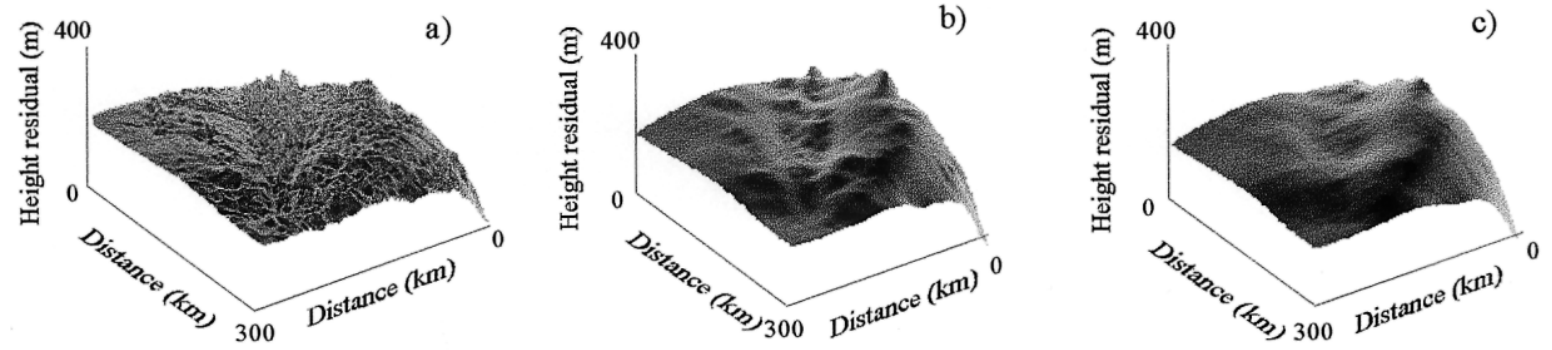

Fig. 6. (a) A shaded isometric view of the Northeast Greenland Ice Stream derived from an unsmoothed $1 \mathrm{~km}$ DEM of the area. The regional slope was removed by fitting a plane to the data to emphasize the morphology of the stream itself. The elevations are therefore residual values, obtained after subtracting the planar surface. (b) The same area and illumination conditions as in ( $a$ ), but for a DEM with $10 Z$ smoothing. (c) The same area and illumination conditions as in (a), but for a DEM with 20Z smoothing. 


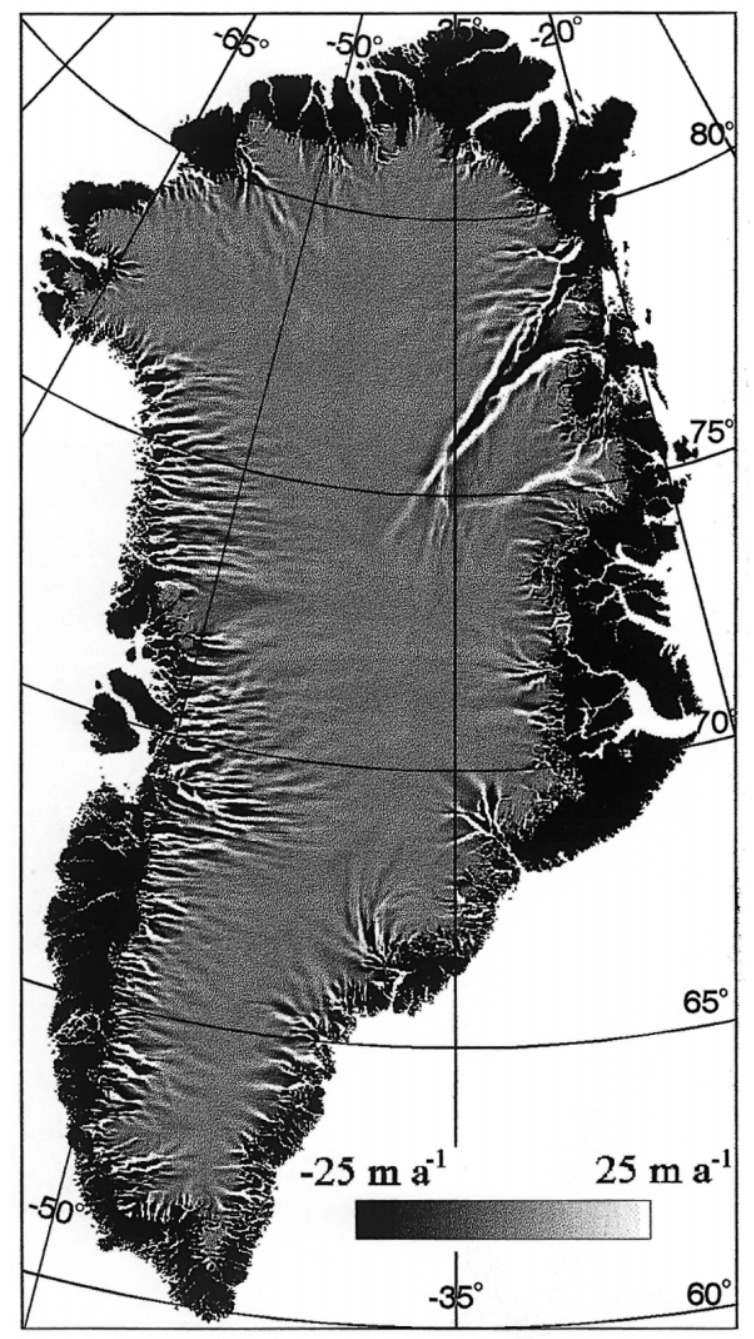

Fig. 8. Spatial pattern of differences $\left(U_{\mathrm{b} 10}-U_{\mathrm{b} 20}\right)$ in balance-velocity estimate for the two smoothing distances used in the finite-difference scheme: $10 Z$ and $20 Z$.

with the use of InSAR velocities derived from a single ascending or descending pair of images. In this case it is necessary to use a DEM (or other information on flow direction) to convert $U_{\text {los }}$ to $U_{\mathrm{ds}}$. If a DEM is used, the conversion implicitly incorporates the shallow-ice approximation. However, the InSAR velocities provide information at much shorter wavelengths than 10-20Z. Thus, locally, in areas where longitudinal stresses are important and where the flow direction deviates from the regional slope aspect, the correction from $U_{\text {los }}$ to $U_{\mathrm{ds}}$ may introduce significant errors into the small-scale velocity field. This problem can be overcome by combining ascending and descending passes (Joughin and others, 1998). This has been achieved in a few cases, but, due to the limited availability and coverage of SAR data, it is often not possible. When considering the regional velocity pattern averaged over distances of about $20 Z$ or more, however, this issue is not relevant.

\section{Humboldt Gletscher region}

In the interior of the ice sheet, where the flow is homogeneous, the accuracy of the input datasets available controls the reliability of $U_{\mathrm{b}}$. Errors in surface slope are insignificant (Bamber and others, 1998), and there is no ablation. The error estimate for these areas is 14\%. Agreement between $U_{\mathrm{b} 20}$ and $U_{\mathrm{ds}}$ in sector 1 is about $5-10 \%$. As the InSAR velocities were fixed using balance velocities for this sector, it is not surprising that there is relatively good agreement. If independent GPS data had been available to provide an absolute velocity reference for the InSAR data, it would have been possible to examine whether steady-state, balanced conditions were being met in this region.

The northern sector (2) displays much poorer agreement. This is due primarily to errors in the ice-thickness data used to calculate the balance velocities near the coast. These values have, in general, been extrapolated from a coarse grid of airborne data (Letréguilly and others, 1991b), and in areas with a large gradient in $Z$ (e.g. near the ice edge and small drainage basins on the east coast) the relative errors in $Z$ can be substantial. Figure $4 d$ shows that the relative difference in velocities increases towards the coast, reaching a maximum at the ice edge. Errors in the InSAR velocity data have been estimated as only $\pm 2.3 \mathrm{~m} \mathrm{a}^{-1}$ for this area, and clearly the balance velocities provide an inaccurate representation due to errors in the input data used to calculate them. Near the margins of the ice sheet (within about $50 \mathrm{~km}$ of the ice edge) ablation rates also need to be estimated, introducing a further level of uncertainty in this region. Furthermore, for surface slopes greater than about $1.0^{\circ}$, errors in the DEM increase substantially (Bamber and others, 1998) and the smoothing distance used becomes particularly important. We therefore believe that in these marginal areas, without better input data, the balance velocities do not provide a reliable proxy for measured values.

\section{GONCLUSIONS}

Balance velocities are clearly a useful way of providing a synoptic view of the depth-averaged velocity field of large ice masses. Agreement between a small number of GPS data was surprisingly good, suggesting that, inland from the margins, balance velocities could provide a reliable and useful constraint in numerical ice-sheet models. Compared with InSAR measurements, they provide a measure of the timeand depth-averaged velocity. The comparisons undertaken in this study have highlighted the respective weaknesses in both types of measurement. In particular, some of the problems related to converting line-of-sight to downslope velocities were noted, especially when short-wavelength flow features are of interest and when using tandem phase ERS SAR data. Balance velocities in the ablation region of the ice sheet are not reliable, although they provide information about the distribution of flow at the margins. Both the method of calculating the balance velocity and the smoothing distance used to remove the effects of longitudinal stresses were important in determining the quality and usefulness of the resultant dataset. This was highlighted by an investigation of balance velocities in the region of the Northeast Greenland Ice Stream.

\section{AGKNOWLEDGEMENTS}

The authors would like to thank R. Warner of the Cooperative Research Centre for the Antarctic and Southern Ocean Environment (Antarctic CRC), Hobart, Australia, for providing the balance-velocity finite-difference code, G. Hamilton of the Byrd Polar Research Center for providing the GPS data and P. Huybrechts for providing the numerical modelling data. R.J.H was funded under U.K. National Environment Research Council grant GR3/9791. The authors are also grateful to the scientific editor and three anonymous referees 
for their valuable comments which led to improvements in this paper.

\section{REFERENCES}

Bamber, J. L. and P. Huybrechts. 1996. Geometric boundary conditions for modelling the velocity field of the Antarctic ice sheet. Ann. Glaciol., 23, 364-373.

Bamber, J. L., S. Ekholm and W. B. Krabill. 1997. A digital elevation model of the Greenland ice sheet and validation with airborne laser altimeter data. In Third ERS Scientific Symposium, 17-21 March 1997, Florence, Italy. Proceedings. Vol. 2. Frascati, Italy, European Space Agency, 843-847. (ESA Publication SP-414.)

Bamber, J. L., S. Ekholm and W. B. Krabill. 1998. The accuracy of satellite radar altimeter data over the Greenland ice sheet determined from airborne laser data. Geophys. Res. Lett., 25(16), 3177-3180.

Budd, W. F. and D. B. Carter. 1971. An analysis of the relation between the surface and bedrock profiles of ice caps. F. Glaciol., 10(59), 197-209.

Budd, W. F. and R. C. Warner. 1996. A computer scheme for rapid calculations of balance-flux distributions. Ann. Glaciol., 23, 21-27.

Ekholm, S. 1996. A full coverage, high resolution topographic model of Greenland computed from a variety of digital elevation data. 7. Geophys. Res., 101 (B10), 21,961-21,972.

Fahnestock, M., R. Bindschadler, R. Kwok and K. Jezek. 1993. Greenland ice sheet surface properties and ice dynamics from ERS-1 SAR imagery. Science, 262(5139), 1530-1534.

Greve, R. 1997. Application of a polythermal three-dimensional ice sheet model to the Greenland ice sheet: response to steady-state and transient climate scenarios. F. Climate, 10(5), 901-918.

Grootes, P. M., M. Stuiver, J.W. C. White, S. Johnsen and J. Jouzel. 1993. Comparison of oxygen isotope records from the GISP2 and GRIP Greenland ice cores. Nature, 366(6455), 552-554.

Hanssen, R., H. Zebker, R. Klees and S. Barlag. 1998. On the use of meteorological observations in SAR interferometry. In IGARSS '98. 18th International Geoscience and Remote Sensing Symposium, 6-10 July 1998, Seattle, Washington. Proceedings. Pisacataway, NJ, Institute of Electrical and Electronics Engineers, 1644-1646.

Huybrechts, P., A. Letréguilly and N. Reeh. 1991. The Greenland ice sheet and greenhouse warming. Global and Planetary Change, 3(4), 399-412.

Joughin, I. R., D. P. Winebrenner and M. A. Fahnestock. 1995. Observations of ice-sheet motion in Greenland using satellite radar interferometry. Geophys. Res. Lett., 22(5), 571-574.

Joughin, I., R. Kwok and M. Fahnestock. 1996a. Estimation of ice-sheet motion using satellite radar interferometry: method and error analysis with application to Humboldt Glacier, Greenland. F. Glaciol., 42(142), 564-575.

Joughin, I., S. Tulaczyk, M. Fahnestock and R. Kwok. 1996b. A mini-surge on the Ryder Glacier, Greenland, observed by satellite radar interferometry. Science, 274(5285), 228-230.

Joughin, I., M. Fahnestock, S. Ekholm and R. Kwok. 1997. Balance velocities of the Greenland ice sheet. Geophys. Res. Lett., 24(23), 3045-3048.

Joughin, I. R., R. Kwok and M. A. Fahnestock. 1998. Interferometric estimation of three-dimensional ice-flow using ascending and descending passes. IEEE Trans. Geosci. Remote Sensing, GE-36(1), 25-37.

Letréguilly, A., N. Reeh and P. Huybrechts. 1991a. The Greenland ice sheet through the last glacial-interglacial cycle. Palaeogeogr., Palaeoclimatol., Palaeoecol., 90(4), 385-394.

Letréguilly, A., P. Huybrechts and N. Reeh. 1991b. Steady-state characteristics of the Greenland ice sheet under different climates. F. Glaciol., 37(125), 149-157.

Mohr, J.J., N. Reeh and S. N. Madsen. 1998. Three-dimensional glacial flow and surface elevation measured with radar interferometry. Nature, 391 (6664), 273-276.

Paterson, W. S. B. 1994. The physics of glaciers. Third edition. Oxford, etc., Elsevier.

Radok, U., R. G. Barry, D. Jenssen, R. A. Keen, G. N. Kiladis and B. McInnes. 1982. Climatic and physical characteristics of the Greenland ice sheet. Part I and II. Boulder, CO, University of Colorado. Cooperative Institute for Research in Environmental Sciences.

Rignot, E., K. C. Jezek and H. G. Sohn. 1995. Ice flow dynamics of the Greenland ice sheet from SAR interferometry. Geophys. Res. Lett., 22(5), 575-578.

Scambos, T. A., M. J. Dutkiewicz, J. C. Wilson and R. A. Bindschadler. 1992. Application of image cross-correlation to the measurement of glacier velocity using satellite image data. Remote Sensing Environ., 42(3), 177-186.

Van der Veen, C. J. and I. M. Whillans. 1990. Flow laws for glacier ice: comparison of numerical predictions and field measurements. 7. Glaciol., 36(124), 324-339. 